\title{
DNA methylation is involved in sexual differentiation and sex chromosome evolution in the dioecious plant garden asparagus
}

\author{
Shu-Fen Li' ${ }^{1}$, Can-Can Lv' ${ }^{1}$ Li-Na Lan ${ }^{1}$, Kai-Lu Jiang ${ }^{1}$, Yu-Lan Zhang ${ }^{1}$, Ning Li ${ }^{1}{ }^{1}$, Chuan-Liang Deng ${ }^{1}$ and
} Wu-Jun Gao ${ }^{1 凶}$

\begin{abstract}
DNA methylation is a crucial regulatory mechanism in many biological processes. However, limited studies have dissected the contribution of DNA methylation to sexual differentiation in dioecious plants. In this study, we investigated the variances in methylation and transcriptional patterns of male and female flowers of garden asparagus. Compared with male flowers, female flowers at the same stages showed higher levels of DNA methylation. Both male and female flowers gained DNA methylation globally from the premeiotic to meiotic stages. Detailed analysis revealed that the increased DNA methylation was largely due to increased $\mathrm{CHH}$ methylation. Correlation analysis of differentially expressed genes and differentially methylated regions suggested that DNA methylation might not have contributed to the expression variation of the sex-determining genes SOFF and TDF1 but probably played important roles in sexual differentiation and flower development of garden asparagus. The upregulated genes AOMS1, AOLAP3, AOAMS, and AoLAP5 with varied methylated $\mathrm{CHH}$ regions might have been involved in sexual differentiation and flower development of garden asparagus. Plant hormone signaling genes and transcription factor genes also participated in sexual differentiation and flower development with potential epigenetic regulation. In addition, the CG and CHG methylation levels in the $Y$ chromosome were notably higher than those in the $X$ chromosome, implying that DNA methylation might have been involved in $Y$ chromosome evolution. These data provide insights into the epigenetic modification of sexual differentiation and flower development and improve our understanding of sex chromosome evolution in garden asparagus.
\end{abstract}

\section{Introduction}

As an essential epigenetic regulatory mechanism in eukaryotic species, DNA methylation contributes greatly to transposon silencing, heterochromatin organization, genome integrity maintenance, and gene expression regulation $^{1-4}$. In higher plant genomes, DNA methylation usually exists in CG, CHG, and CHH (where $\mathrm{H}$ represents A, T, or C) sequence contexts ${ }^{5}$. The genomes of different plants show distinct DNA methylation profiles ${ }^{6}$. Moreover, the DNA methylation ratio and level change dynamically in different plant tissues at diverse developmental stages ${ }^{7,8}$.

Correspondence: Wu-Jun Gao (gaowujun@htu.cn)

${ }^{1}$ College of Life Sciences, Henan Normal University, Xinxiang 453007, China
Accumulating reports have suggested that DNA methylation is a key modulator of numerous aspects of plant development, such as fruit ripening ${ }^{7,9}$, seed germination and development ${ }^{8}$, and stress responses ${ }^{10}$. However, the possible role of DNA methylation in sexual differentiation and unisexual flower development in dioecious plants is largely unclear.

The dioecious system rarely occurs in flowering plants, in contrast to the phenomenon in animals. Only approximately $6 \%$ of angiosperm species are dioecious; that is, each individual harbors only male or female flowers ${ }^{11}$. Dioecious plants are believed to have evolved from hermaphroditic species numerous times ${ }^{12,13}$. Most dioecious plants carry sex chromosomes, which are well documented

\section{(c) The Author(s) 2021}

(c) (i) Open Access This article is licensed under a Creative Commons Attribution 4.0 International License, which permits use, sharing, adaptation, distribution and reproduction c. in any medium or format, as long as you give appropriate credit to the original author(s) and the source, provide a link to the Creative Commons license, and indicate if changes were made. The images or other third party material in this article are included in the article's Creative Commons license, unless indicated otherwise in a credit line to the material. If material is not included in the article's Creative Commons license and your intended use is not permitted by statutory regulation or exceeds the permitted use, you will need to obtain permission directly from the copyright holder. To view a copy of this license, visit http://creativecommons.org/licenses/by/4.0/. 
to be derived from autosomes. Sex chromosome evolution process involves several developmental events, such as recombination suppression, repeat sequence accumulation, and Y chromosome heterochromatization and degenera$\operatorname{tion}^{14}$. It has been revealed that DNA methylation may play a role in sex chromosome degeneration ${ }^{15}$. The determination and differentiation of opposite sexes are usually genetically governed by sex-determining genes located on sex chromosomes ${ }^{14}$. The sexes of plants are usually reflected in the flowers, which are formed during the reproductive stage. Sex determination/differentiation and flower development processes are regulated by dynamic networks among a variety of genes, transcription factors (TFs), and other modulators, including microRNAs and epigenetic modifications ${ }^{16-18}$. Recent studies have identified several sex-linked candidates sex-determining genes in a few dioecious plants ${ }^{19-22}$. The potential functions of these genes vary dramatically due to the independent origins of dioecious plants. Moreover, many sexbiased genes involved in sexual differentiation and flower development have been detected by using comparative transcriptome sequencing and other traditional methods $^{16,23-25}$.

Garden asparagus (Asparagus officinalis) is a dioecious plant species, and its sex is controlled by $\mathrm{X}$ and $\mathrm{Y}$ sex chromosomes. It has a $1 \mathrm{C}$ genome size of $1.3 \mathrm{~Gb}$ and $2 n=2 x=20$ chromosomes $^{26,27}$. Genome sequencing of garden asparagus has enabled assembly of $986 \mathrm{Mb}$ of the genome, including a small male-specific region of the $\mathrm{Y}$ chromosome (MSY, $847 \mathrm{~kb})^{20}$. Two genes located in MSY have been detected as candidates for sex determination: one is a potential female suppression gene $(S O F F)$, and the other (TDF1) may function as a male activator ${ }^{20,28}$. In addition, a number of sex-biased genes have been detected and are potentially involved in sexual differentiation and flower development processes ${ }^{24,25}$.

The present study aimed to determine whether DNA methylation influences the transcriptional levels of sexbiased genes and whether it is involved in sex determination, sexual differentiation, and flower development processes of garden asparagus. A detailed analysis of the garden asparagus methylome and transcriptome was carried out using male and female flowers at different developmental stages. The results can provide valuable information for understanding the DNA methylation landscape and epigenetic regulation of sexual differentiation and flower development in garden asparagus.

\section{Materials and methods}

\section{Plant material and sample preparation}

Plants of the garden asparagus variety 'UC309' were grown in the experimental plot of Henan Normal University. The sex of the garden asparagus was determined by the floral phenotype. The individuals used in this study were progenies of one male and one female plant. The flower buds of males (six plants) and females (six plants) at the premeiotic $(0.5-0.7 \mathrm{~mm}$ in length) and meiotic $(1.0-1.6 \mathrm{~mm}$ in length) stages were separately pooled and immediately frozen in liquid nitrogen. The premeiotic and meiotic stages were determined by referring to published articles $^{25,29}$ and cytogenetic analysis. Genomic DNA from flower buds was isolated using the traditional cetyltrimethylammonium bromide method. Total RNA from asparagus flower buds (three biological replicates per sample) was isolated using TRIzol reagent (Life Technologies, CA, USA).

\section{Whole-genome bisulfite sequencing (BS-seq)}

After DNA concentration and integrity were detected, the DNA libraries for BS-seq were prepared from $500 \mathrm{ng}$ of genomic DNA, which was sonicated into $100-300 \mathrm{bp}$ fragments. The fragmented DNA was purified and then subjected to end-blunting, A-extension, and adaptor ligation. For bisulfite treatment, an EZ DNA MethylationGold $^{\text {TM }}$ Kit (Zymo, CA, USA) was used. The converted DNA fragments were subsequently amplified and then subjected to sequencing via the Illumina $\mathrm{HiSeq}^{\mathrm{TM}} 2500$ platform.

\section{DNA methylation data analysis}

The clean reads were mapped to the garden asparagus genome with the BSMAP program (version 2.90) ${ }^{30}$ using the default settings. Thereafter, a custom Perl script was used to identify methylated cytosines, and the methylated cytosines were tested as described in Lister et $\mathrm{al}^{31}$.

Differentially methylated regions (DMRs) between different comparison groups were identified using Pearson's chi-square test $\left(\chi^{2}\right)$ in methylKit (version 1.7.10) ${ }^{32}$. We first partitioned the genome into $200 \mathrm{bp}$ regions with overlaps of $100 \mathrm{bp}$. Then, DMRs $(F D R \leq 0.05)$ were identified with methylation differences of $0.25,0.25$, and 0.15 for $\mathrm{CG}, \mathrm{CHG}$, and $\mathrm{CHH}$, respectively.

\section{RNA-seq and data analysis}

RNA-seq was conducted as previously described ${ }^{25}$. After sequencing and filtering, the clean reads were rRNA-depleted using Bowtie $2^{33}$ and then mapped to the garden asparagus genome with TopHat2 (version 2.0.3.12) ${ }^{34}$ with the parameter settings - 1 -no-coveragesearch -r 50 -mate-std-dev 80. Transcript abundance quantification was performed by using RSEM ${ }^{35}$. Differentially expressed genes (DEGs) of different comparison groups were determined with the criteria of a foldchange $\geq 2$ and a $q$ value $<0.05$.

\section{DNA methylation and gene expression correlation analysis}

The annotated genes were first classified into four groups on the basis of gene expression levels: 
nonexpressed (none) (FPKM value $\leq 1$ ), weakly expressed (low) $(1<\mathrm{FPKM} \leq 10)$, moderately expressed (middle) $(10<$ FPKM $\leq 100)$, and highly expressed (high) (FPKM > $100)$ genes. The methylation levels in the gene body and adjacent regions of these groups were determined. DMRassociated DEGs were analyzed by identifying DEGs harboring DMRs in the three contexts within the promoter, gene body, and downstream $2 \mathrm{~kb}$ regions.

\section{Results}

DNA methylation landscape of garden asparagus flower buds

We conducted a whole-genome BS-seq of premeiotic and meiotic flower buds of males and females (Fig. 1a). A total of 1.05 billion clean single-end $150 \mathrm{bp}$ reads were generated, and BS-seq had an average sequencing depth of $29.06 \times$ per sample. On average, approximately $73.6 \%$ of cytosines of the garden asparagus reference genome sequence were covered by one or more reads (Supplemental Fig. 1).

A total of $\sim 314$ and 312 million mCs in male premeiotic and meiotic flower buds and $~ 317$ and 310 million $\mathrm{mCs}$ in female premeiotic and meiotic flower buds were identified. After at least four-read coverage filtering, 145.8 million methylated cytosine sites in each sample were identified on average, accounting for $46.54 \%$ of the cytosines with the reference genome. Generally, the methylation patterns among the four samples were similar (Fig. 1b). We used male premeiotic stage flower buds as representative samples for methylation pattern analysis. As shown in Fig. 1c, the CG and CGH methylation sites were highly abundant in gene-poor heterochromatin regions with high transposable element (TE) frequencies, whereas $\mathrm{CHH}$ methylation displayed a weak and uniform distribution, which is consistent with observations in other plant species ${ }^{36}$.

The mean methylation levels of CG, CHG, $\mathrm{CHH}$, and total $\mathrm{C}$ were $87.41 \%, 72.75 \%, 8.90 \%$, and $29.45 \%$, respectively (Fig. 1b, Supplemental Table 1). Most CG and CHG sites were heavily methylated. In contrast, the majority of $\mathrm{CHH}$ sites showed relatively low methylation levels, with $80 \%$ of $\mathrm{CHH}$ sites presenting methylation levels of 20-60\% (Supplemental Fig. 2).

\section{DNA methylation profiles in genes and TEs}

The methylation profiles in different genomic regions were examined separately. For the genic regions, the CG sites showed the highest methylation in the gene body, decreased methylation in gene-adjacent regions, and the lowest methylation in transcriptional start/end sites (TSS/ TES) (Fig. 1d). Methylation in the CHG context showed moderate levels in the gene body, increased levels in upstream regions, and slightly lower levels in downstream regions (Fig. 1d). The $\mathrm{CHH}$ context showed hypomethylation in gene bodies but increased methylation levels in flanking sequences (Fig. 1d). For all three sequence contexts, exons presented hypomethylation compared with introns (Supplemental Fig. 3). To further investigate whether DNA methylation affects the gene transcriptional levels, we clustered genes into four groups on the basis of their transcriptional levels. The data showed that CG methylation in gene bodies was positively correlated with gene transcriptional levels, while CG methylation around TSS/TES and downstream $2 \mathrm{~kb}$ regions decreased gradually with increasing gene expression levels. For the $\mathrm{CHG}$ and $\mathrm{CHH}$ contexts, the methylation levels in TSS/TES and downstream $2 \mathrm{~kb}$ regions were negatively correlated with transcriptional levels. However, $\mathrm{CHH}$ methylation in promoter regions and gene transcription levels showed a positive correlation (Supplemental Fig. 4).

Consistent with reports in most other plant species, all three cytosine contexts showed hypermethylation in TE regions but low methylation in the flanking regions (Fig. 1e). The hypermethylation of TEs is possibly responsible for the suppression of abundant TEs in the garden asparagus genome.

\section{Comparative analysis of DNA methylation in male and female flowers at different developmental stages}

In general, female flower buds showed higher methylation levels than male flower buds in all contexts (CG, $\mathrm{CHG}$, and $\mathrm{CHH}$ ), with more remarkable differences in the CHH context (Supplemental Table 1, Fig. 1b). During asparagus flower development, the methylation levels in the meiotic stage tended to be higher than those in the premeiotic stage in both male and female flower buds. Such differences were mild at CG and CHG sites but more significant in the asymmetric $\mathrm{CHH}$ context (Supplemental Table 1, Fig. 1b). Accordingly, the highest methylation levels were observed in female flower buds at the meiotic stage in both genes and TEs. In contrast, male flower buds at the premeiotic stage showed the lowest methylation levels. Detailed examination revealed that the high DNA methylation levels were primarily due to increased $\mathrm{CHH}$ methylation (Fig. 1d, e).

To investigate the methylation differences more specifically, we identified DMRs in a pairwise fashion (F_pre versus M_pre, F_mei versus M_mei, M_pre versus M_mei, and F_pre versus F_mei). At the premeiotic stage, we identified a total of 9969, 12,275, and 15,796 DMRs in the CG, CHG, and $\mathrm{CHH}$ contexts, respectively, in female flowers relative to male flowers. Among these DMRs, 15,202 were hypermethylated (hyper-DMRs), while 22,838 were hypomethylated (hypo-DMRs) (Fig. 2a). We discovered that $41.5 \%$ of the total DMRs were in $\mathrm{CHH}$ sites, while CHG DMRs and CG DMRs exhibited lower percentages (Fig. 2b). The meiotic stage (F_mei vs. 


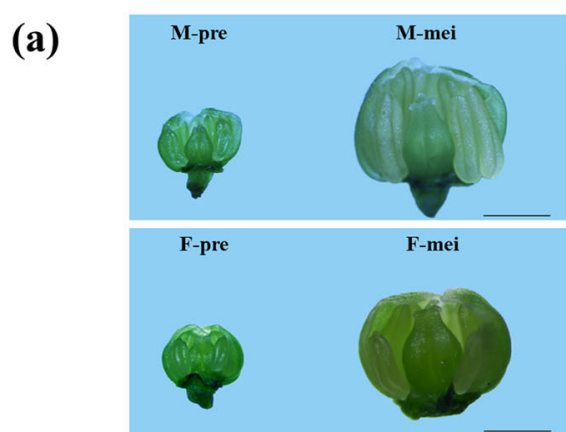

(b)

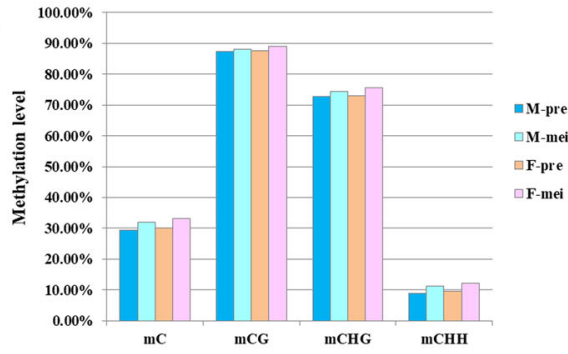

(d)

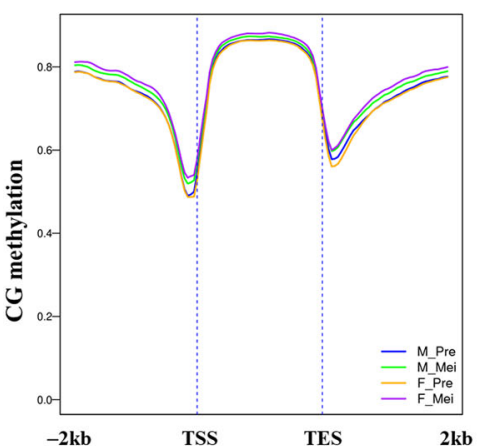

(e)

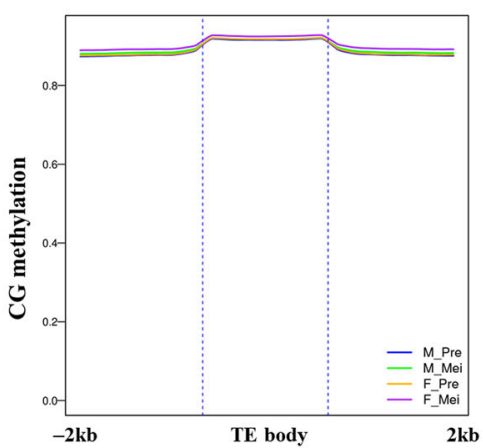

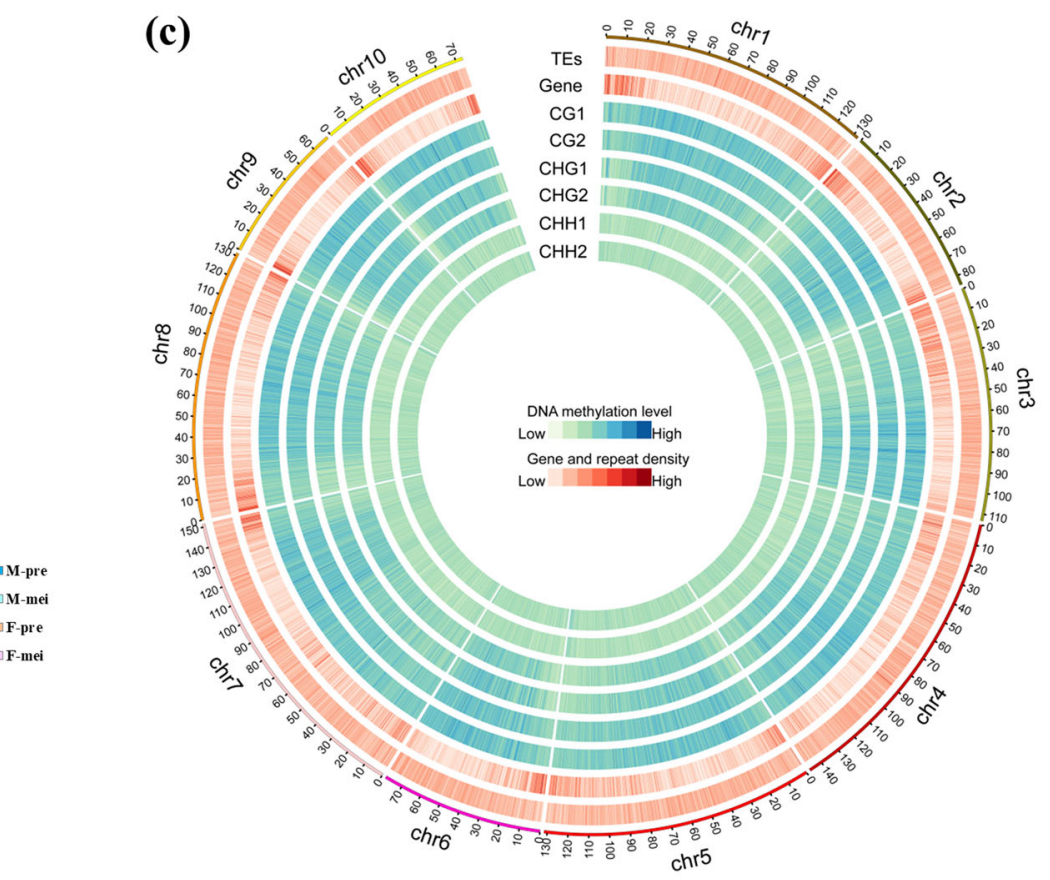

(c)
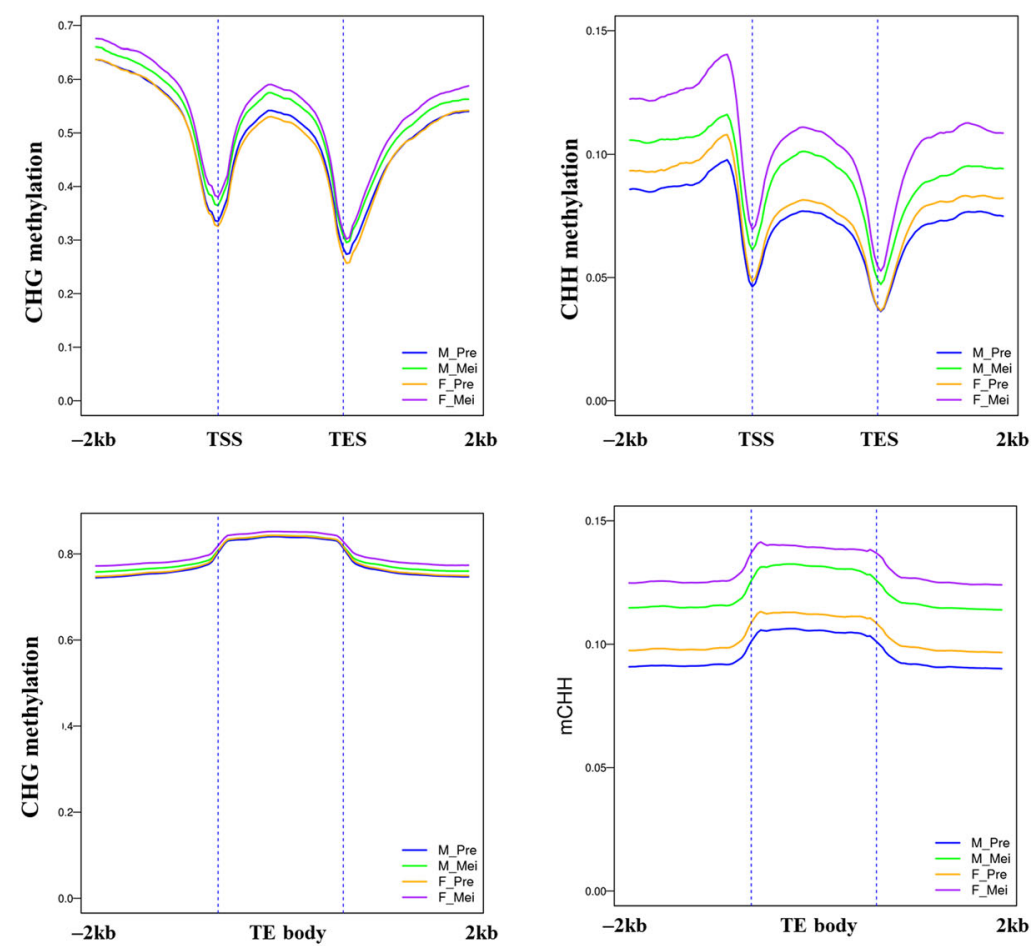

Fig. 1 DNA methylation characterization of garden asparagus. a Morphological comparison of male and female flower buds of garden asparagus at different developmental stages. b Mean DNA methylation levels of C, CG, CHG, and CHH in different samples. c Overview of DNA methylation in 10 chromosomes. $\mathbf{d}$ DNA methylation profiles in gene bodies and adjacent regions in the three sequence contexts. e DNA methylation characteristics of TEs and upstream/downstream regions in each context

M_mei) showed similar trends. More strikingly, $\mathrm{CHH}-$ DMRs were more abundant in the meiotic stage (60.4\%) than in the premeiotic stage, and hypo-CHH-DMRs were the most abundant (Fig. 2a, b). For the developmental stages, pairwise comparisons of M_pre vs. M_mei and F_pre vs. F_mei yielded similar results, with much more 

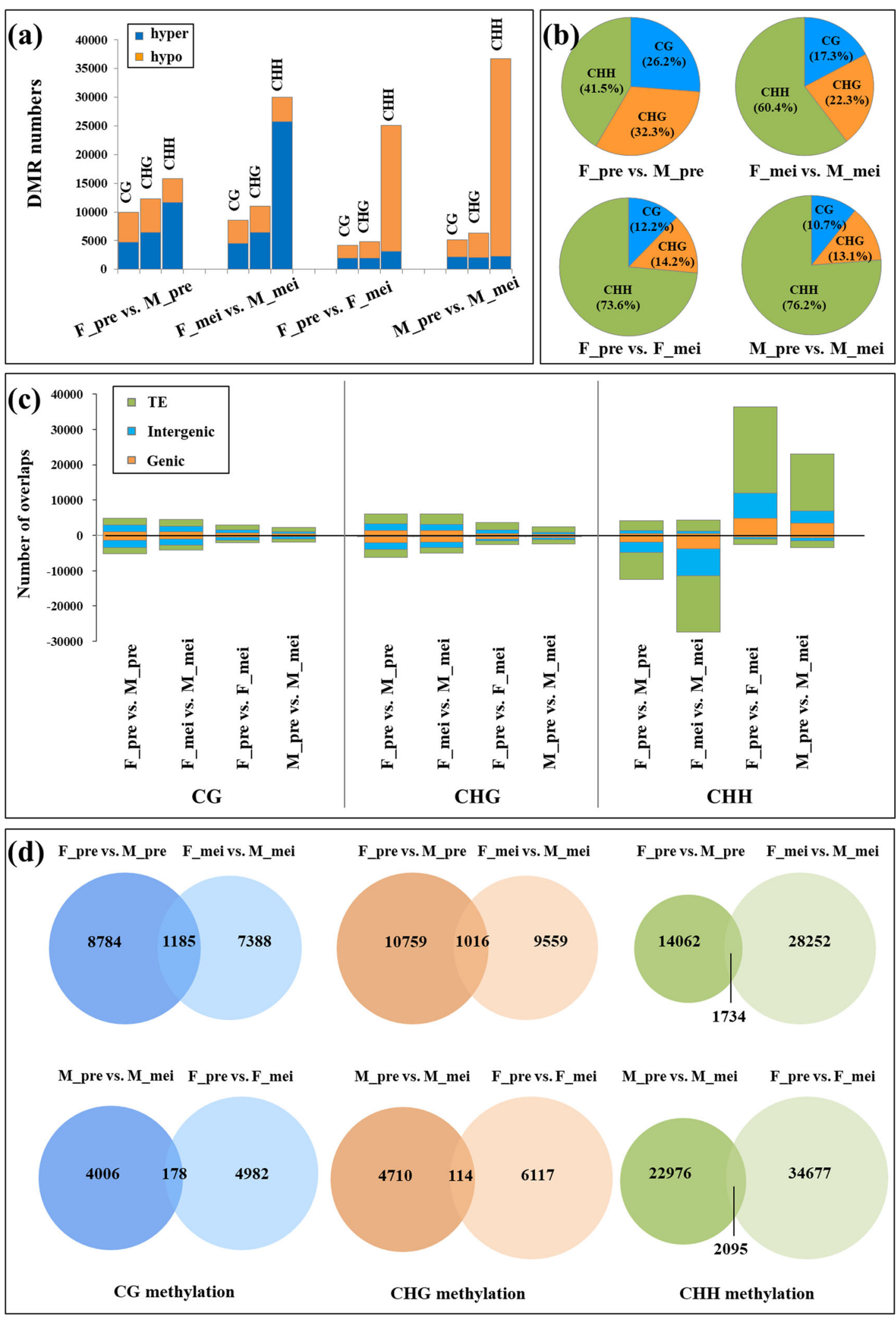

Fig. 2 (See legend on next page.) 
(see figure on previous page)

Fig. 2 DMRs in male and female flower buds at the premeiotic and meiotic stages. a DMRs were identified by paired comparisons of the BS-seq patterns of the four samples (male flower buds at the premeiotic stage, M_pre; female flower buds at the meiotic stage, F_pre; male flower buds at the meiotic stage, M_mei, and female flower buds at the meiotic stage, M_mei) in each context. The DMR numbers, including the hyper- and hypoDMRs for each cytosine context, are shown. b DMR proportions for all three contexts. $\mathbf{c}$ Localization of DMRs in different annotated features, including genic, TE, and intergenic regions. d DMRs were largely nonoverlapping in pairwise comparisons between F_pre vs. M_pre and F_mei vs. M_mei (upper). The DMRs between M_pre vs. M_mei and F_pre vs. F_mei also did not overlap (lower)

hyper-DMRs than hypo-DMRs, and CHH-DMRs were predominant (Fig. 2a, b).

We further assessed the genomic locations of these DMRs and observed that the DMRs were predominantly located in TEs and intergenic regions for all three contexts (Fig. 2c). In addition, although the variation patterns between F_pre vs. M_pre and F_mei vs. M_mei were similar, the DMRs of all three contexts did not overlap. A similar tendency was observed between the pairwise comparisons of M_pre vs. M_mei and F_pre vs. F_mei (Fig. 2d). These results indicate that dynamic methylation patterns are potentially responsible for garden asparagus sexual differentiation and distinct flower development.

\section{Comparison of the transcriptomes between male and female asparagus flowers at different developmental stages}

We generated transcriptome profiles for the same male and female flower buds to investigate the potential transcriptional consequences of methylation variations related to garden asparagus male and female flower development. Clustering analysis showed consistency among the three replicates of each sample (Fig. 3a). In particular, the flowers at the premeiotic stage from both males and females clustered together, which was consistent with their similar phenotypes (Fig. 3a). Accordingly, there were fewer sex-biased DEGs at the premeiotic stage than at the meiotic stage (Fig. 3b). The numbers of upregulated DEGs at both the premeiotic and meiotic stages were higher than the number of downregulated DEGs in males compared with females. With regard to male and female flower development, 4701 and 2708 DEGs were identified for males and females, respectively. Most of these DEGs were upregulated at the meiotic stage (Fig. 3b). Moreover, using criteria of an FPKM $<0.4$ in one sex and an FPKM $>$ 2 in the other sex, we identified 39 male-specific and 37 female-specific genes at the premeiotic stage. More sexspecific genes were detected at the meiotic stage, with 738 being male-specific and 189 being female-specific.

\section{DEG-DMR correlation analysis of male and female flowers at the meiotic stage}

To investigate whether the sexual differentiation process in garden asparagus was associated with DNA methylation, we focused on analyzing DMR-related genes by comparing F_mei and M_mei flowers because the meiotic stage is the critical stage of sexual differentiation ${ }^{24,29}$. Approximately 12,999 hyper-DMRs and 36,635 hypo-DMRs were identified in M_mei flowers compared with F_mei flowers. Among them, 17,667 were distributed in the gene body or promoter/ downstream regions and thus potentially contributed to gene regulation during sexual differentiation and flower development. A total of 8,199 genes were detected as sexbiased DMR-related genes in garden asparagus flower buds. Among them, 5960 were female hyper-DMR-associated genes, while 2,239 were male hyper-DMR-associated genes at the meiotic stage. Among the female hyper-DMRassociated genes, we identified 639 female-biased DEGs (cluster 1), 702 male-biased DEGs (cluster 2), and 4,619 nonDEGs (cluster 3). Among the male hyper-DMR-associated genes, a total of 208 female-biased DEGs (cluster 4), 284 male-biased DEGs (cluster 5), and 1,747 non-DEGs (cluster 6) were identified. Cluster 1 and cluster 4 genes were highly expressed in female flowers. In contrast, cluster 2 and cluster 5 genes presented higher transcriptional levels in male flowers. However, the transcriptional levels of the cluster 3 and cluster 6 genes did not show significant differences (Supplemental Fig. 5). These data suggested that the majority of DMRs did not have a significant impact on the transcriptional levels of adjacent genes.

We further analyzed the DMR-associated DEGs. The orthologs of the known genes involved in the anther development pathway were examined, and six genes were identified as male-biased genes, including EMS1, AMS, MS1, MS2, LAP3, and LAP5 (Fig. 4a). Among these genes, two were DMR-associated DEGs. LAP3 harbors a hypomethylated $\mathrm{CHH}$ region in the promoter region, and $M S 1$ has a $\mathrm{CHH}$ hypomethylation region in the downstream region. In the plant hormone signal transduction pathway, 38 genes showed sex-biased expression at the meiotic stage. Among them, 11 DEGs showed varied DNA methylation (Fig. 4b). These genes mainly belonged to auxin- and cytokinin-related signal transduction pathways. Specifically, the cytokinin-related signaling genes AoARR2 and AoARR3 and two AoARR17 genes were more highly expressed in male flower buds than in female flower buds. Most of these genes presented hypomethylation at $\mathrm{CHH}$ sites in male flowers in comparison with female flowers (Fig. 4b).

In addition, the DMR-associated DEGs comprised numerous TF-encoded genes. Among the 224 DEGs between the two types of flowers at the meiotic stage, approximately $35.3 \%$ (79) were correlated with DMRs. 

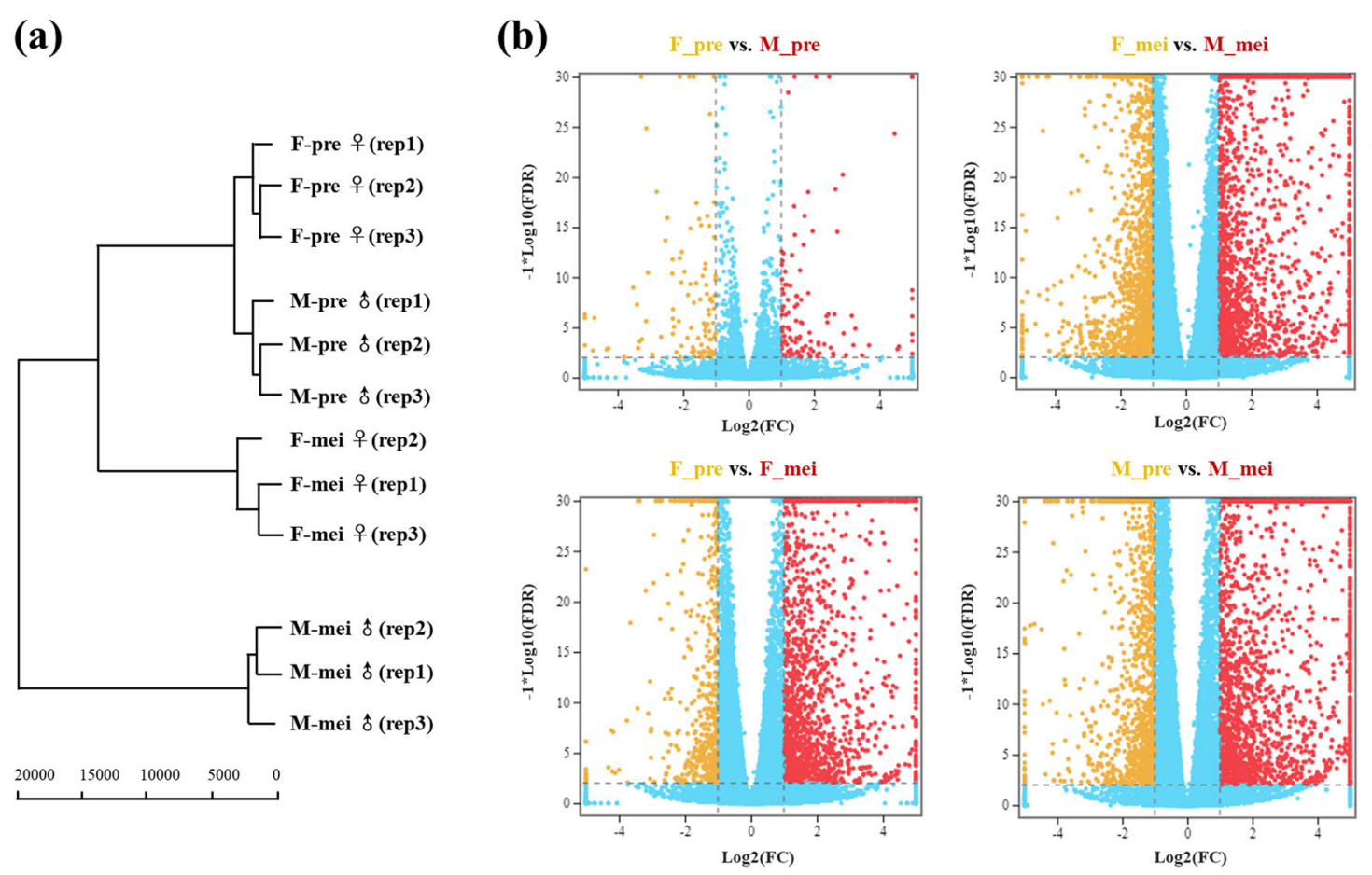

Fig. 3 Clustering and comparison of DEGs in male and female flower buds of garden asparagus at different developmental stages. a Clustering of male and female flower samples at different developmental stages (based on 22,322 expressed genes). b Gene expression comparisons of different groups: F_pre vs. M_pre, F_mei vs. M_mei, F_pre vs. F_mei, and M_pre vs. M_mei

Of these DMR-associated DEGs, 40 were preferentially expressed in males, and 39 were female-biased expressed genes. The differentially expressed TFs included members of the bHLH, MYB, NAC, MADS, bZIP, and other TF families. Members of the MYB and NAC families were highly enriched among male-biased DEGs, whereas members of the bHLH family were highly enriched in female-biased DEGs (Supplemental Fig. 6). Most of these genes $(74.7 \%)$ showed differential methylation in the $\mathrm{CHH}$ context, and the $\mathrm{CHH}$ context DMRs were mainly hypomethylated in males. Interestingly, most CG-type DMRs (76.9\%) were located in the gene body, whereas the majority of the $\mathrm{CHH}$ context DMRs (78\%) were located in promoter or downstream gene regions (Fig. 4c). These results demonstrated that methylation dynamics at $\mathrm{CHH}$ sites might contribute to the sexual differentiation of garden asparagus.

\section{Genes potentially modulated by DNA methylation variance during unisexual flower development}

To demonstrate the role of DNA methylation-related gene modulation in flower development, we conducted interrelation analysis between DEGs and DMRs for the M_pre vs. M_mei and F_pre vs. F_mei comparison groups. During male flower development, two malebiased DEGs within the anther development pathway showed varied DNA methylation. AoAMS harbored a hypermethylated $\mathrm{CHH}$ DMR in the promoter region at the meiotic stage in comparison with the premeiotic stage. The other gene, AoLAP5, had a hypermethylated $\mathrm{CHH}$ DMR in the gene body (Fig. 5). In addition, a number of DMR-associated DEGs implicated in plant hormone signal transduction and TFs were identified in the M_pre vs. M_mei (Supplemental Figs. 7 and 8) and F_pre vs. F_mei comparison groups (Supplemental Fig. 9). The majority of these genes presented hypermethylation in the $\mathrm{CHH}$ context in meiotic flowers compared with premeiotic flowers (Supplemental Figs. 8 and 9).

\section{DNA methylation did not have a significant influence on} the expression variance of sex-determining genes

The Y chromosome present in males is a key determinant of sex. The MSY of garden asparagus has 13 genes. Among these genes, SOFF was found to suppress female function, and TDF1 was regarded as a male promoter. Thus, the two genes were considered to be critical sexdetermining genes. They specifically existed in the male genome and were absent from the female genome. SOFF was not expressed in male flowers at the premeiotic stage and was weakly expressed at the meiotic stage. TDF1 was mildly expressed at the premeiotic stage in male flowers but was significantly upregulated at the meiotic stage. 


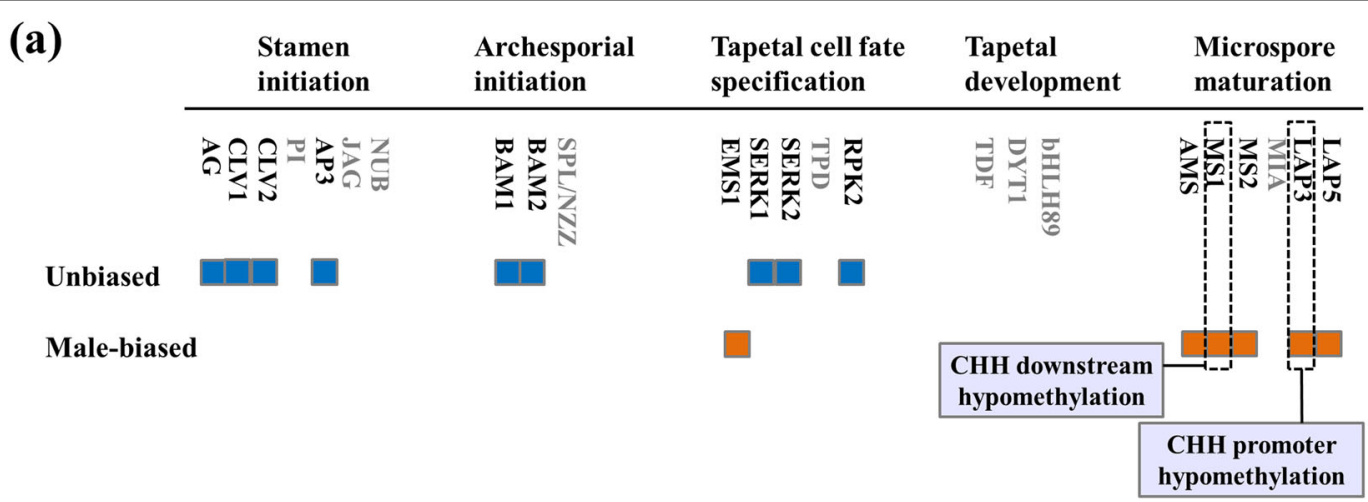

(b)

F_mei vs. M_mei

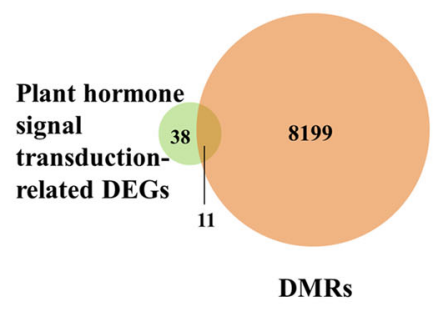

(c)

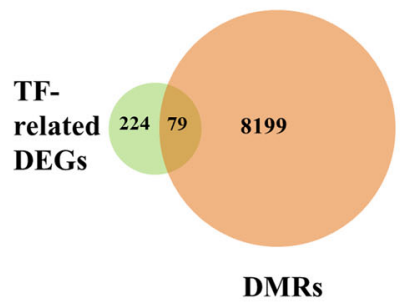

Promoter

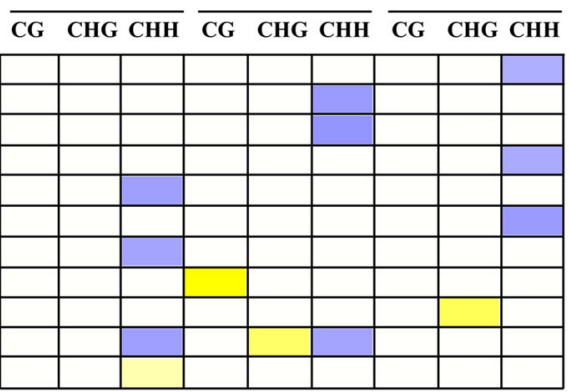

$\begin{array}{cc}-40 & 40\end{array}$
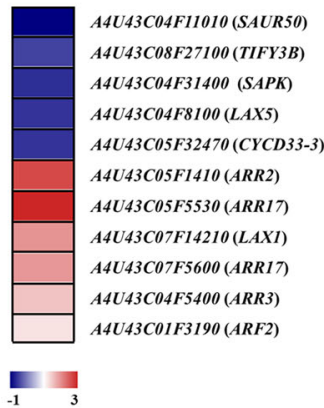

Female-biased DEGs

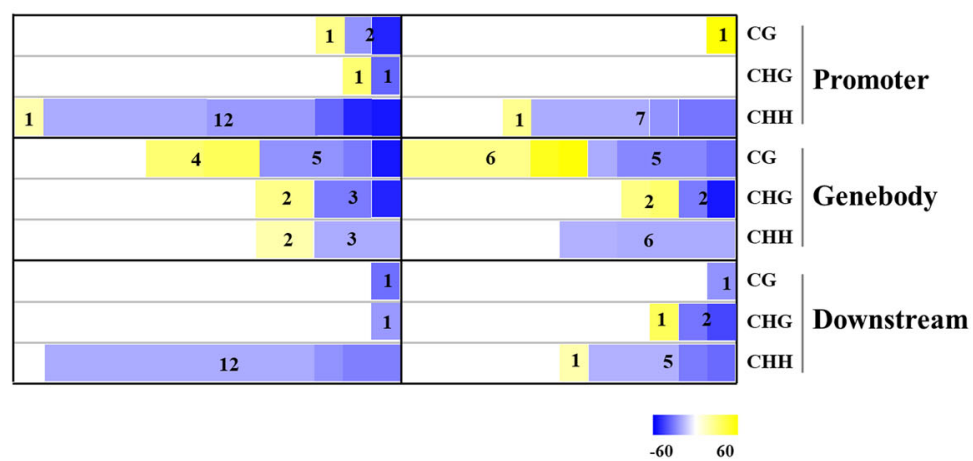

Fig. 4 Association analysis of DEG-DMR correlated genes between male and female flowers at the meiotic stage. a Expression patterns and DEG-DMR association analysis of critical genes involved in the anther development pathway between male and female flowers at the meiotic stage. Genes whose names are in a gray color were unable to be successfully annotated in our transcriptome data. Genes with no sex-biased expression are presented with blue squares, whereas genes showing male-biased expression are presented with orange squares. The two male-biased genes marked with dashed square frames showed marked differential methylation between the two opposite sexes. $\mathbf{b}$ Number of DMR-associated DEGs belonging to the plant hormone signaling pathway (left) and the differential methylation profiles of male- and female-biased genes involved in the plant hormone signaling pathway (right). c Number of DMR-associated DEGs encoding TFs (left) and the differential methylation profiles of male- and female-biased genes annotated as TF genes (right)

However, the methylation statuses of the two genes and flanking sequences did not differ between the two developmental stages (Supplemental Fig. 10). Thus, we speculated that DNA methylation might not directly influence the expression variances of sex-determining genes of garden asparagus.

\section{DNA methylation profiling in sex chromosomes}

To investigate the methylation profiles of sex chromosomes of garden asparagus, we explored the methylation levels of $\mathrm{X}$ and $\mathrm{Y}$ chromosomes in flower buds (Fig. 6). We found that $\mathrm{CpG}$ and $\mathrm{CHG}$ methylation levels were apparently elevated in the majority of $\mathrm{Y}$ chromosomes 


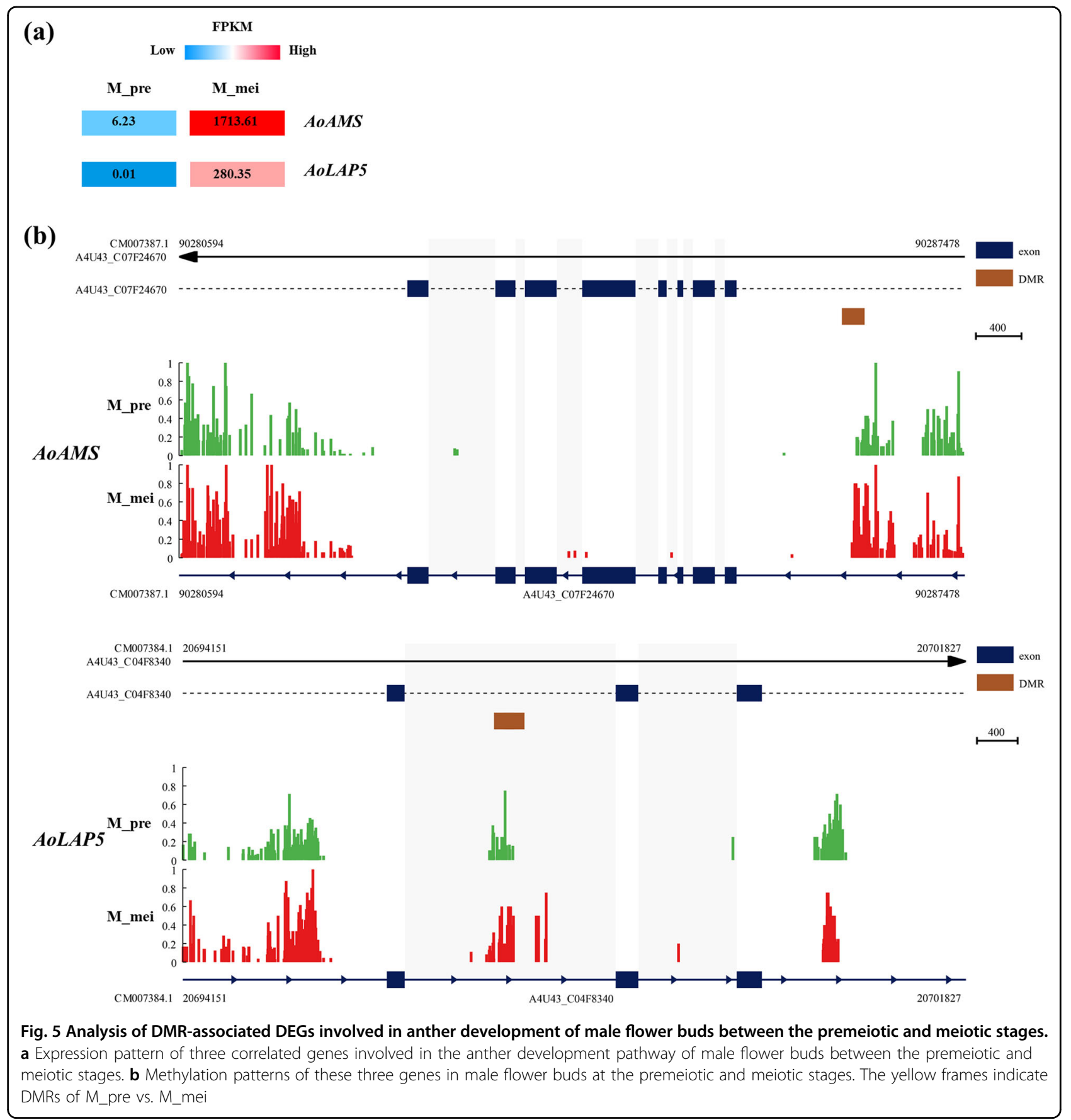

compared with $\mathrm{X}$ chromosomes; that is, the $\mathrm{Y}$ chromosome was hypermethylated in the CpG and CHG contexts. In contrast, the $\mathrm{CHH}$ methylation level of the $\mathrm{X}$ chromosome was higher than that of the $\mathrm{Y}$ chromosome. In addition, the methylation distribution and levels of the $\mathrm{X}$ chromosome in females and males were nearly identical in all three contexts.

DMR profiling of sex chromosomes showed that $\mathrm{Y}$ chromosomes had wider-ranging methylation variances than $\mathrm{X}$ chromosomes. The DMRs in the $\mathrm{X}$ chromosomes showed differences between males and females, but no consistent patterns were found.

\section{Discussion}

\section{Sex-biased DNA methylation in garden asparagus}

Whole-genome BS-seq and subsequent comparison analyses were performed to investigate sex-biased DNA methylation in garden asparagus. Interestingly, most discovered DMRs were found in the $\mathrm{CHH}$ context, and the majority of DMRs were located in TE regions. 
(a)

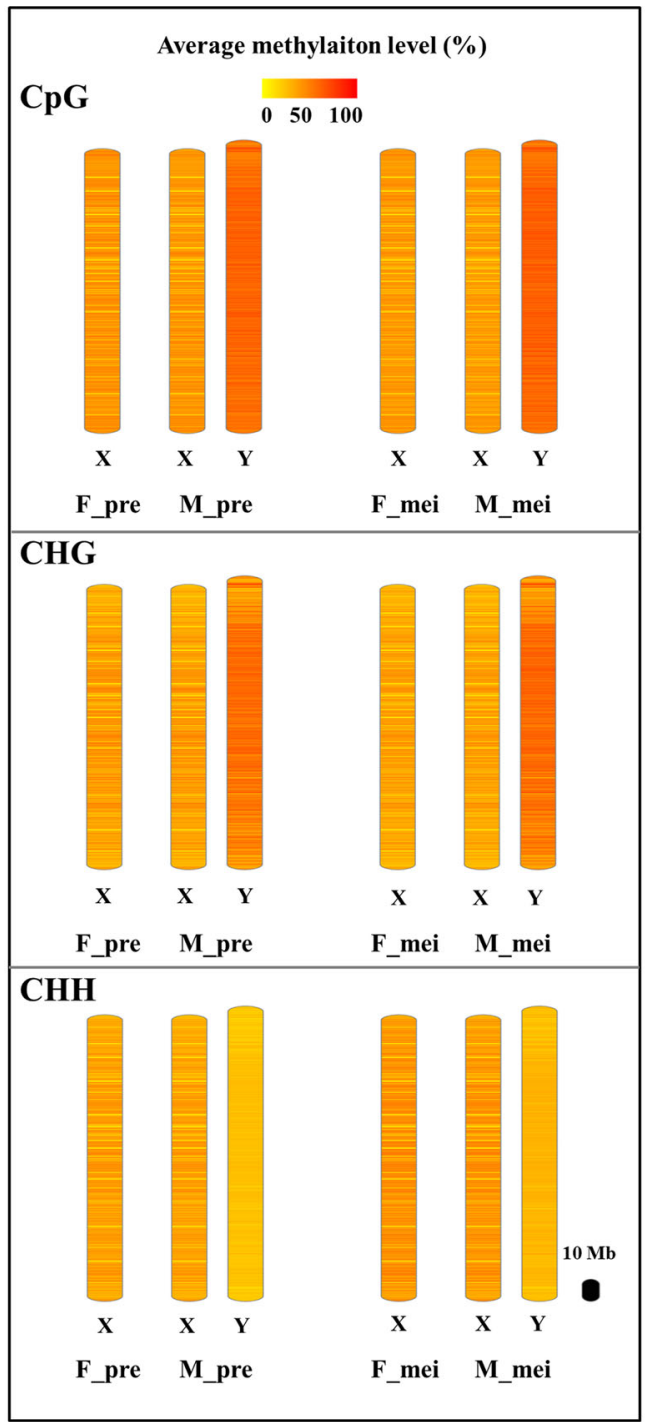

(b)

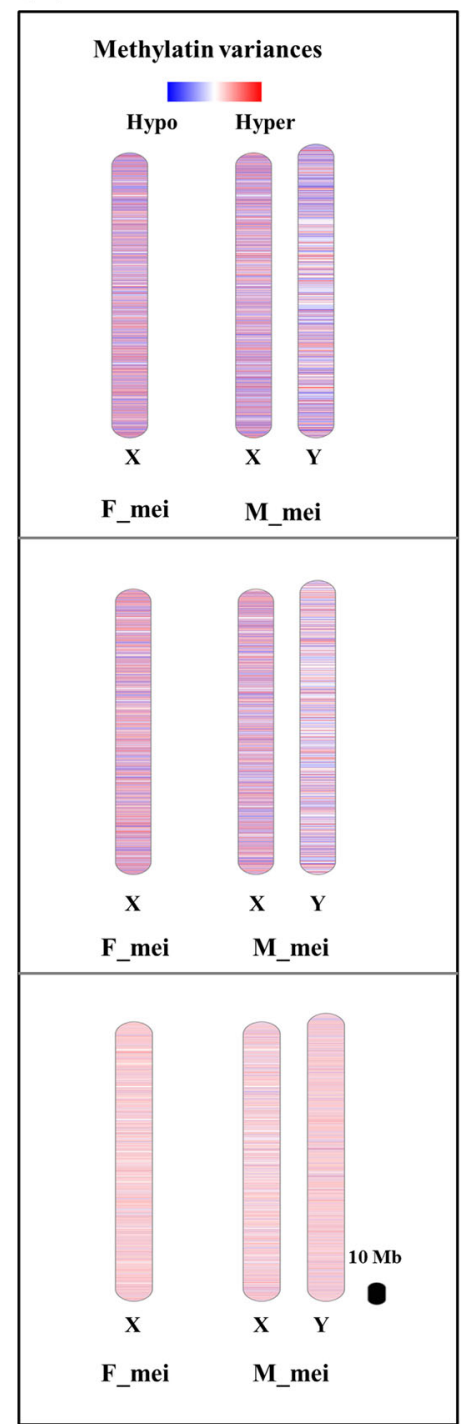

Fig. 6 Methylation profiles of garden asparagus sex chromosomes. a Average methylation levels of the $X$ and $Y$ chromosomes in male and female flowers. $\mathbf{b}$ DMR distribution of male and female flowers at the meiotic stage

The existing sex-specific DMRs reflect DNA methylation variances between male and female flowers. These sexspecific methylation variations might function in shaping sex characteristics by regulating gene transcription levels. However, we discovered that the majority of genes carrying DMRs in their gene bodies or flanking regions did not present significant changes in their expression levels. In fact, this phenomenon has also been discovered in other studies on the relationships of DNA methylation with a number of biological processes ${ }^{37-39}$. For example, one study investigated the influence of epigenetic modification on soybean domestication and evolution by comparing and analyzing the variation in DNA methylation in several soybean cultivars and related materials.
A total of 5,412 DMRs were identified; however, codifferential expression analyses suggested that merely $22.54 \%$ of DMRs were associated with varied gene expression levels ${ }^{39}$. Interpretation of these results is complicated because although tremendous advancements in DNA methylation have been achieved in recent years, much DNA methylation remains enigmatic.

\section{DNA methylation may not be involved in shaping the} expression profiles of sex-determining genes in garden asparagus

Previous studies have revealed that DNA methylation may participate in sex determination in several dioecious plants. Treatment with the hypomethylating chemical 
5-azacytidine in Silene induces a sex alteration in some male individuals, suggesting that the sex-determining process involves DNA methylation in Silene ${ }^{40}$. In hexaploid persimmon (Diospyros kaki), male buds have a higher methylated promoter of MeGI than female buds; $M e G I$ is autosomal gene-regulating another fertility and can be targeted by the sex-determining gene OGI. DNA methylation inhibition induces the conversion of immature male buds into female flowers ${ }^{41}$. Aside from these findings, epigenetic modulation of sex determination has not been discovered in other dioecious plants. Dioecy has independently evolved many times in plants. Thus, the sex determination mechanisms are distinct in different plants, even in closely related species. For example, the epigenetic sex regulation mechanism in hexaploid persimmon is remarkably divergent from the genetic determination of its close relative diploid persimmon ${ }^{41}$. In garden asparagus, during male flower development, the expression levels of the two sex-determining genes were increased, whereas the DNA methylation levels of the two genes and adjacent regions were not significantly changed. The results indicate that DNA methylation may not have strong influences on the transcriptional variances of sexdetermining genes of garden asparagus. It is well known that sex is stable in garden asparagus and little affected by external factors. This is in agreement with the fact that sex determination of garden asparagus may not involve epigenetic regulation. However, our knowledge of the molecular mechanisms of these sex-determining genes in garden asparagus is rather limited. We look forward to further functional studies elucidating the modulation mechanisms of these sex-determining genes and confirming the relationship between DNA methylation and sex determination.

\section{DNA methylation and sex chromosome evolution of garden asparagus}

Sex chromosomes evolved from pairs of autosomes through a gradual and continuous process including a number of key events, such as sex-determining gene formation, recombination suppression, repetitive sequence accumulation, and $\mathrm{Y}$ chromosome degeneration $^{14,42}$. DNA methylation has been considered a crucial modulator involved in the whole evolutionary process of sex chromosomes ${ }^{43}$. However, few studies have been conducted to support this view ${ }^{44}$. Garden asparagus harbors "young" homomorphic sex chromosomes, which are suitable for the investigation of the events and mechanisms of early sex chromosome evolution ${ }^{45}$. During the sex chromosome evolution process, repetitive sequence accumulation and heterochromatization are believed to have facilitated suppression of recombination and sex chromosome differentiation ${ }^{46}$. In fact, DNA methylation represents a defense mechanism to suppress TEs and other types of invasive $\mathrm{DNA}^{47,48}$. DNA methylation facilitates the shaping of heterochromatin ${ }^{49,50}$, and it is regarded to contribute to recombination suppression of sex chromosomes in papaya ${ }^{51}$. As has been reported previously in Silene latifolia, which has large heteromorphic Y chromosomes, TE accumulation may lead to hypermethylation of the $\mathrm{Y}$ chromosome ${ }^{15}$. In this study, we found that the methylation levels in the CpG and CHG contexts of $\mathrm{Y}$ chromosomes were apparently higher than those of $\mathrm{X}$ chromosomes. These results indicated that DNA methylation might function in suppressing recombination between the sex-specific regions of the $\mathrm{X}$ and $\mathrm{Y}$ chromosomes in garden asparagus.

\section{Possible epigenetic regulation of genes involved in the anther development pathway and their possible roles in sexual differentiation and male flower development in garden asparagus}

Flowering, the most essential developmental process in higher plant life, consists of a complicated network involving a diverse range of gene interactions ${ }^{52,53}$. In dioecious plants, sex-determining genes may modulate downstream genetic networks and other pathways to mediate unisexual flower development ${ }^{54}$. We compared the methylation levels and gene expression in male and female flower buds of garden asparagus at the premeiotic and meiotic stages. At the premeiotic stage, the male and female flower buds showed no morphological differences. Comparative transcriptome analysis also presented a small number of DEGs. However, the sex-determining gene TDF1 was expressed, albeit at a low level. The results suggested that sexual differentiation was initiated at this stage. At the meiotic stage, a large number of DEGs were identified. The sex-determining gene TDF1 showed much higher expression at the meiotic stage than at the premeiotic stage, and SOFF also showed a higher expression level, implying that the meiotic stage is a critical sexual differentiation stage. Among the DMR-associated DEGs between females and males at the meiotic stage, MS1 and $L A P 3$ are critical genes involved in the anther development pathway of Arabidopsis thaliana. In addition, association analysis between male flowers at different stages showed that AMS and LAP5 were involved in the anther development pathway. Thus, epigenetic regulation of MS1, LAP3, AMS, and LAP5 might mediate garden asparagus flower development and sexual differentiation. MS1 encodes a protein similar to the PHD-finger motif $\mathrm{TF}$ and plays a significant role in male gametogenesis and another development. Homozygous $m s 1$ mutants of $A$. thaliana fail to produce functional pollen ${ }^{55}$. AMS encodes a bHLH-type TF that is essential for male fertility. It can also bind to the promoter regions of a set of anther development genes and regulate their expression ${ }^{56}$. The other two genes (LAP3 and LAP5) encode 
calcium-dependent phosphotriesterase superfamily proteins and chalcone/stilbene synthase family proteins, respectively. They are both involved in pollen exine formation $^{57,58}$. Thus far, no studies have reported the epigenetic regulation of these four genes. However, many genes participating in the anther development pathway are mediated by DNA methylation ${ }^{59,60}$. Interestingly, we found that these genes with higher expression in male flowers at the meiotic stage than in female flowers at the meiotic stage or male flowers at the premeiotic stage all harbored hypomethylation in the $\mathrm{CHH}$ context. These results indicate that the functions of AoMS1, AoLAP3, AoAMS, and AoLAP5 in garden asparagus sexual differentiation and flower development potentially involve epigenetic regulation.

\section{Plant hormone signaling genes and TF genes under epigenetic regulation are likely associated with sexual differentiation and flower development in garden asparagus}

Plant hormones are essential regulators that exert profound and diverse effects on almost the entire plant development process ${ }^{61}$. Reports have shown that plant hormone signaling genes are involved in the modulation of sex determination and sexual differentiation of several dioecious plants ${ }^{22,62}$. In Actinidia, the cytokinin response modulator SyGI, which acts as a dominant suppressor of female development, has been identified as a potential sex determinant gene ${ }^{22}$. Importantly, DNA methylation has been found to contribute to the plant hormone signaling process of sexual expression in papaya ${ }^{38}$. In this study, we found that male and female flowers exhibited obvious methylation variance in the number of DEGs involved in plant hormone signal transduction. Most of these DEGs belonged to cytokinin- and auxin-response pathways. In particular, AoARR2, AoARR3, and two AoARR17 genes showed higher expression in male flower buds than in female flower buds. The $A R R$ gene family is related to cytokinin signaling ${ }^{63}$. It is known that cytokinin signaling can interact with typical MADS-box proteins to influence early inflorescence development ${ }^{64}$. Studies in Arabidopsis have shown that AtARR16 coupled with AtAHP6 may restrict cytokinin signaling and participate in gynoecium development ${ }^{65}$. PbRR9, a homologous gene of AtARR16 and AtARR 17 at the sex-determining region, has been proposed to have an epigenetically mediated role in sex determination in Populus balsamifera ${ }^{62}$. The high expression levels of $A o A R R$ genes in male flowers might contribute to inflorescence differentiation and androecium formation in garden asparagus. This is in contrast with the situation in papaya, in which a CPARR5 gene associated with hypomethylation at its downstream region is downregulated in male flowers. These results demonstrate that the mechanisms of sexual differentiation differ in different dioecious plants. However, these results confirm that plant hormone-mediated transcription regulation associated with epigenetic modulation indeed plays a role in the sexual differentiation and flower development of these dioecious plants.

TFs are the main regulators of sexual differentiation and flower development processes in plants. Indeed, some genes within the anther development pathway and the plant hormone signaling process discussed above encode TFs. For example, AMS is a member of the bHLH TF family. In addition, a number of co-differentially expressed TF genes with DMRs were identified by comparing male and female flowers in this study. Among the various TF families, MYB and NAC were enriched in male flowers, whereas bHLH was enriched in female flowers. Numerous studies have shown that epigenetic regulation of TF genes plays important role in sex differentiation in animals ${ }^{66,67}$. For example, in zebrafish, elevated temperature induces masculinization by reducing the expression levels of two TF genes, sox $9 b$, and esr1, via DNA methylation ${ }^{67}$. Epigenetic regulation of TF genes has also been confirmed to participate in sex determination/differentiation and flower development in some plant species $^{68,69}$. It has been reported that epigenetic changes in the promoter of $C m W I P 1$, a $\mathrm{C} 2 \mathrm{H} 2$ zinc-finger TF gene in melon, can repress the transcription of CmACS7, inhibit gynoecium development and lead to staminate flowers $^{70}$. In Arabidopsis, the MADS-box gene FLF (for FLOWERING LOCUS F) can repress flowering regulated by vernalization and DNA methylation ${ }^{71}$. We speculate that the identified co-differentially expressed TF genes with DMRs might be associated with garden asparagus sexual differentiation and unisexual flower development. The characterization and future analysis of key genes that participate in plant hormone signaling and genes encoding TFs will be helpful in understanding the complicated network of sexual differentiation in garden asparagus.

\section{Acknowledgements \\ This work was financially supported by grants from the National Natural Science Foundation of China (31970240 and 31470334) and the Natural Science Foundation of Henan Province (202300410242).}

\section{Author contributions}

W.J.G. and S.F.L. conceived and supervised the project. C.C.L., L.N.L. and K.L.J. analyzed the data. Y.L.Z. and N.L. designed and drew the figures. S.F.L. drafted the manuscript. W.J.G. and C.L.D. revised the paper.

\section{Data availability}

All the raw sequencing data reported in this paper have been deposited in the Genome Sequence Archive under accession number CRA004058 and are publicly accessible at https://bigd.big.ac.cn/gsa.

Conflict of interest

The authors declare no competing interests. 
Supplementary information The online version contains supplementary material available at https://doi.org/10.1038/s41438-021-00633-9.

Received: 29 March 2021 Revised: 25 May 2021 Accepted: 1 June 2021 Published online: 01 September 2021

\section{References}

1. Panda, K. et al. Full-length autonomous transposable elements are preferentially targeted by expression-dependent forms of RNA-directed DNA methylation. Genome Biol. 17, 170 (2016).

2. Meng, $\mathrm{H}$. et al. DNA methylation, its mediators and genome integrity. Int. J. Biol. Sci. 11, 604-617 (2015).

3. Li, X. et al. High-resolution mapping of epigenetic modifications of the rice genome uncovers interplay between DNA methylation, histone methylation, and gene expression. Plant Cell 20, 259-276 (2008).

4. Wang, $\mathrm{H}$. et al. CG gene body DNA methylation changes and evolution of duplicated genes in cassava. Proc. Natl Acad. Sci. USA 112, 13729-13734 (2015).

5. Zemach, A., McDaniel, I. E., Silva, P. \& Zilberman, D. Genome-wide evolutionary analysis of eukaryotic DNA methylation. Science 328, 916-919 (2010).

6. Niederhuth, C. E., Bewick, A. J., Ji, L., Alabady, M. S. \& Schmitz, R. J. Widespread natural variation of DNA methylation within angiosperms. Genome Biol. 17, 194 (2016).

7. Cheng, J. et al. Downregulation of RdDM during strawberry fruit ripening. Genome Biol. 19, 212 (2018).

8. An, Y. C. et al. Dynamic changes of genome-wide DNA methylation during soybean seed development. Sci. Rep. 7, 12263 (2017).

9. Huang, $\mathrm{H}$. et al. Global increase in DNA methylation during orange fruit development and ripening. Proc. Natl Acad. Sci. USA 116 1430-1436 (2019)

10. Yaish, M. W., Al-Lawati, A., Al-Harrasi, I. \& Patankar, H. V. Genome-wide DNA methylation analysis in response to salinity in the model plant caliph medic (Medicage truncatula). BMC Genomics 19, 78 (2018).

11. Renner, S. S. The relative and absolute frequencies of angiosperm sexual systems: dioecy, monocy, gynodioecy, and un updated online database. Am. J. Bot. 101, 1588-1596 (2014).

12. Bachtrog, D. et al. Sex determination: why so many ways of doing it? PLoS One 12, e1001899 (2014)

13. Ming, R., Wang, J., Moore, P. H. \& Paterson, A. H. Sex chromosomes in flowering plants. Am. J. Bot. 94, 141-150 (2007).

14. Li, S. F., Zhang, G. J., Yuan, J. H., Deng, C. L. \& Gao, W. J. Repetitive sequences and epigenetic modification: inseparable partners play important roles in the evolution of plant sex chromosomes. Planta 243, 1083-1095 (2016)

15. Lorenzo, J. L. R., Hobza, R. \& Vyskot, B. DNA methylation and genetic degeneration of the $Y$ chromosome in the dioecious plant Silene latifolia. BMC Genomics 19, 540 (2018)

16. Chawla, A. et al. Sex-biased temporal gene expression in male and female floral buds of seabuckthorn (Hippophae rhamnoides). PLoS One 10, e0124890 (2015).

17. Diggle, P. K. et al. Multiple developmental processes underlie sex differentiation in angiosperms. Trends Genet. 27, 368-376 (2011).

18. Boualem, A. et al. A cucurbit androecy gene reveals how unisexual flowers develop and dioecy emerges. Science 350, 688-691 (2015).

19. Akagi, T., Henry, I., Tao, R. \& Comai, L. A Y-chromosome-encoded small RNA acts as a sex determinant in persimmons. Science 346, 646-650 (2014).

20. Harkess, A. et al. The asparagus genome sheds light on the origin and evolution of a young Y chromosome. Nat. Commun. 8, 1279 (2017).

21. Torres, M. F. et al. Genus-wide sequencing supports a two-locus model for sexdetermination in Phoenix. Nat. Commun. 9, 3969 (2018).

22. Akagi, T. et al. A Y-encoded suppressor of feminization arose via lineagespecific duplication of a cytokinin response regulator in kiwifruit. Plant Cell 30, 780-795 (2018).

23. Urasaki, N. et al. Digital transcriptome analysis of putative sex-determination genes in papaya (Carica papaya). PloS One 7, e40904 (2012).

24. Harkess, A. et al. Sex-biased gene expression in dioecious garden asparagus (Asparagus officinalis). N. Phytol. 207, 883-892 (2015).

25. Li, S. F. et al. Comparative transcriptome analysis reveals differentially expressed genes associated with sex expression in garden asparagus (Asparagus officinalis). BMC Plant Biol. 17, 143 (2017).
26. Arumuganathan, K. \& Earle, E. D. Nuclear DNA content of some important plant species. Plant Mol. Biol. Rep. 9, 208-218 (1991).

27. Štajner, N., Bohanec, B. \& Javornik, B. Genetic variability of economically important Asparagus species as revealed by genome size analysis and rDNA ITS polymorphisms. Plant Sci. 162, 931-937 (2002).

28. Harkess, A. et al. Sex determination by two Y-linked genes in garden asparagus. Plant Cell 32, 1790-1796 (2020).

29. Caporali, E., Carboni, A. \& Galli, M. Development of male and female flowers in Asparagus officinalis. Search for point of transition from hermaphroditic to unisexual developmental pathway. Sex. Plant Reprod. 7, 239-249 (1994).

30. Xi, Y. \& Li, W. BSMAP: whole genome bisulfite sequence MAPping program. BMC Bioinforma. 10, 232 (2009)

31. Lister, R. et al. Human DNA methylomes at base resolution show widespread epigenomic differences. Nature 462, 315 (2009).

32. Akalin, A. et al. MethyKit: a comprehensive $\mathrm{R}$ package for the analysis of genome-wide DNA methylation profiles. Genome Biol. 13, R87 (2012).

33. Langmead, B. \& Salzberg, S. L. Fast gapped-read alignment with Bowtie 2. Nat Methods 9, 357-359 (2012)

34. Kim, D. et al. TopHat2: accurate alignment of transcriptomes in the presence of insertions, deletions and gene fusions. Genome Biol. 14, R36 (2013).

35. Li, B. \& Dewey, C. N. RSEM: accurate transcript quantification from RNA-Seq data with or without a reference genome. BMC Bioinforma. 12, 1 (2011).

36. Zhang, J. et al. Autotetraploid rice methylome analysis reveals methylation variation of transposable elements and their effects on gene expression. Proc. Natl Acad. Sci. USA 112, E7022-E7029 (2015).

37. Zhou, P. et al. DNA methylome and transcriptome landscapes revealed differential characteristics of dioecious flowers in papaya. Hortic. Res. 7, 81 (2020).

38. Rajkumar, M. S., Shankar, R., Garg, R. \& Jain, M. Bisulphite sequencing reveals dynamic DNA methylation under desiccation and salinity stresses in rice cultivars. Genomics 112, 3537-3548 (2020).

39. Shen, Y. et al. DNA methylation footprints during soybean domestication and improvement. Genome Biol. 19, 128 (2018).

40. Janoršek, B., Široký, J. \& Vyskot, B. Epigenetic control of sexual phenotype in a dioecious plant, Melandrium album. Mol. Gen. Genet. 250, 483-490 (1996).

41. Akagi, T., Henry, I. M., Kawai, T., Comai, L. \& Tao, R. Epigenetic regulation of the sex determination gene MeGl in polyploid persimmon. Plant Cell 28, 2905-2915 (2016).

42. Abbott, J. K, Nordén, A. K. \& Hansson, B. Sex chromosome evolution: historical insights and future perspectives. Proc. R. Soc. B 284, 20162806 (2017).

43. Gorelick, R. Evolution of dioecy and sex chromosomes via methylation driving Muller' ratchet. Biol. J. Linn. Soc. 80, 353-368 (2003).

44. Metzger, D. C. H. \& Schulte, P. M. The DNA methylation landscape of stickleback reveals patterns of sex chromosome evolution and effects of environmental salinity. Genome Biol. Evol. 10, 775-785 (2018).

45. Charlesworth, D. Plant contributions to our understanding of sex chromosome evolution. N. Phytol. 208, 52-65 (2015).

46. de Bello Cioffi, M. et al. The key role of repeated DNAs in sex chromosome evolution in two fish species with ZW sex chromosome system. Mol. Cytogenet. 5, 28 (2012).

47. Noshay, J. M. et al. Monitoring the interplay between transposable element families and DNA methylation in maize. PLoS Genet. 15, e1008291 (2019).

48. Zhou, W., Liang, G., Molloy, P. L. \& Jones, P. A. DNA methylation enables transposable element-driven genome expansion. Proc. Natl Acad. Sci. USA 117 19359-19366 (2020)

49. Mirouze, M. et al. Loss of DNA methylation affects the recombination land scape in Arabidopsis. Proc. Natl Acad. Sci. USA 109, 5880-5885 (2012).

50. Yelina, N. E. et al. DNA methylation epigenetically silences crossover hot spots and controls chromosomal domains of meiotic recombination in Arabidopsis. Genes Dev. 29, 2183-2202 (2015).

51. Zhang, W., Wang, X., Yu, Q., Ming, R. \& Jiang, J. DNA methylation and heterochromatinization in the male-specific region of the primitive $Y$ chromosome of papaya. Genome Res. 18, 1938-1943 (2008).

52. $\mathrm{Li}, \mathrm{H}$. et al. Time-series transcriptome provides insights into the gene regulation network involved in the volatile terpenoid metabolism during the flower development of lavender. BMC Plant Biol. 19, 313 (2019).

53. Wellmer, F. \& Riechmann, J. L. Gene networks controlling the initiation of flower development. Trends Genet. 26, 519-527 (2010).

54. Li, N. et al. Comparative transcriptome analysis of male and female flowers in Spinacia oleracea L. BMC Genomics 21, 850 (2020).

55. Wilson, Z. A., Morroll, S. M., Dawson, J., Swarup, R. \& Tighe, P. J. The Arabidopsis MALE STERILITY1 (MS1) gene is a transcriptional regulator of male 
gametogenesis, with homology to the PHD-finger family of transcription factors. Plant J. 28, 27-39 (2001).

56. $\mathrm{Xu}$, J. et al. The ABORTED MICROSPORES regulatory network is required for postmeiotic male reproductive development in Arabidopsis thaliana. Plant Cell 22, 91-107 (2010).

57. Dobritsa, A. A. et al. LAP3, a novel plant protein required for pollen development, is essential for proper exine formation. Sex. Plant Reprod. 22, 167 (2009).

58. Dobritsa, A. A. et al. LAP5 and LAP6 encode anther-specific proteins with similarity to chalcone synthase essential for pollen exine development in Arabidopsis. Plant Physiol. 153, 937-955 (2010).

59. Yang, $\mathrm{H}$. et al. Whole-genome DNA methylation patterns and complex associations with gene structure and expression during flower development in Arabidopsis. Plant J. 81, 268-281 (2015).

60. Jacobsen, S. E., Sakai, H., Finnegan, E. J., Cao, X. \& Meyerowitz, E. M. Ectopic hypermethylation of flower-specific genes in Arabidopsis. Curr. Biol. 10 179-186 (2000).

61. Gray, W. M. Hormonal regulation of plant growth and development. PLoS Biol. 2, e311 (2004)

62. Bräutigam, K. et al. Sexual epigenetics: gender-specific methylation of a gene in the sex determining region of Populus balsamifera. Sci. Rep. 7, 45388 (2017)

63. Xie, M. et al. A B-ARR-mediated cytokinin transcriptional network directs hormone cross-regulation and shoot development. Nat. Commun. 9, 1604 (2018).
64. Han, Y., Yang, H. \& Jiao, Y. Regulation of infloresence architecture by cytokinins. Front. Plant Sci. 5, 669 (2014).

65. Reyes-Olaldem, J. I. et al. The bHLH transcription factor SPATULA enables cytokinin signaling, and both activate auxin biosysthesis and transport genes at the medial domain of the gynoecium. PLoS Genet. 13, e1006726 (2017).

66. Nishino, K., Hattori, N., Tanaka, S. \& Shiota, K. DNA methylation-mediated control of Sry gene expression in mouse gonadal development. J. Biol. Chem. 279, 22306-22313 (2004)

67. Han, J. et al. High temperature induced masculinization of zebrafish by downregulation of sox9b and esr 1 via DNA methylation. J. Environ. Sci. 107, 160-170 (2021).

68. Guo, S. et al. Co-ordination of flower development through epigenetic regulation in two model species: rice and Arabidopsis. Plant Cell Physiol. 56, 830-842 (2015)

69. Pelayo, M. A., Yamaguchi, N. \& Ito, T. One factor, many systems: the floral homeotic protein AGAMOUS and its epigenetic regulatory mechanisms. Curr. Opin. Plant Biol. 61, 102009 (2021).

70. Martin, A. et al. A transposon-induced epigenetic change leads to sex determination in melon. Nature 461, 1135-1138 (2009).

71. Sheldon, C. C. et al. The FLF MADS box gene: a repressor of flowering in Arabidopsis regulated by vernalization and methylation. Plant Cell 11, 445-458 (1999). 